\title{
Effects of Inclination angle and Free Convection on Velocity Profile for a Steady 2-Dimensional Magnetohydrodynamic Fluid Flow in an Inclined Cylindrical Pipe
}

\author{
B. Odongo, R. Opiyo, and A. Manyonge,
}

\begin{abstract}
Effects of inclination and free convection on velocity profile for magnetohydrodynamic (MHD) fluid flow in an inclined cylindrical pipe has been investigated. The governing partial differential equations are the equations of continuity, momentum and energy which are converted into ordinary differential equation by employing similarity transformation and solved numerically by the Runge- Kutta fourth order scheme with shooting method. The findings, which are presented in the form of tables and graphs reveal that; when Hartmann number, Grashoff number and Gamma are decreased, the velocity of the fluid increases. The results of the study may be useful for the different model investigations, especially, in various areas of science and technology in which optimal inclination and free convection are utilized.
\end{abstract}

Index Terms-MHD, Free convection, Shooting method, Nondimensionalisation, Runge-Kutta method.

\section{INTRODUCTION}

$\mathbf{M}$ HD flow through pipes has its relevance in many engineering problems such as MHD power generators, cooling system with liquid metals, petroleum industries, geothermal energy extractions and many others. Shateyi and Marewo[13] obtained a numerical solution for Lamina flow along a heated stretching cylinder at the boundary. They used method of Successive Relaxation and plotted velocity and temperature fields for values of curvature parameter, permeability parameter, Prandtl number and Nusselt number. Shooting method solution for magnetohydrodynamic at the boundary flow along a stretching cylinder was obtained by Swati[15]. He presented solutions for stretching cylinders without varying the magnetic field but with slip conditions partially at the boundary. The graphs plotted showed that there is a decrease in velocity as the velocity slip and magnetic parameters increase. Gasljevic K. et al[6] investigated the buoyancy effects on heat transfer and temperature profile in horizontal pipe flow of drag reducing fluids. Opiyo R. et al [12] obtained a numerical solution of a steady free convective magnetohydrodynamic flow with consideration of heat and

DOI: http://dx.doi.org/10.24018/ejers.2021.6.7.2665

Published on December 10, 2021.

B.A. Odongo, Department of Pure and Applied Mathematics, Maseno University,Kenya. (e-mail: benardodongo84@yahoo.com).

R. O. Opiyo, Department of Pure and Applied Mathematics, Maseno University, Kenya. (e-mail: richardopiyo08@gmail.com).

A. W. Manyonge, Department of Pure and Applied Mathematics, Maseno University, Kenya. (e-mail: wmanyonge@gmail.com). mass transfer along infinite sinusoidal inclined plate at the surface boundary. Graphical out puts of the solutions from a MATHEMATICA code is presented.

\section{A. Problem Formulation}

Consider an axisymmetric, incompressible, steady laminar flow of an electrically conducting fluid moving along an infinite cylindrical pipe of radius a, inclined with acute angle $\gamma$ from the vertical. The cylindrical coordinates $(r, \theta, z)$, where $\mathrm{r}$ and $\mathrm{z}$ are the radial and axial axes of the cylinder, as in the Figure 1. We also assumed a uniform magnetic field of strength $\left(B_{o}\right)$ with very low Reynolds number $(\mathrm{Re})$ of about 2000 , thus the induced magnetic field can be neglected. It is assumed that the surface temperature of the pipe is $T_{w}$ and the temperature far from the surface is $T_{\infty}$ where $T_{w}>T_{\infty}$. The buoyancy force is produced by the temperature difference between the pipe wall temperature and the temperature of the fluid i.e $T-T_{w}$, under the gravitational force $(g)$. The pressure gradient and the external forces are supposed to be zero, and considering the efforts of Shateyi and Marewo [13], the fundamental Continuity, momentum and energy equations are written respectively as follows:

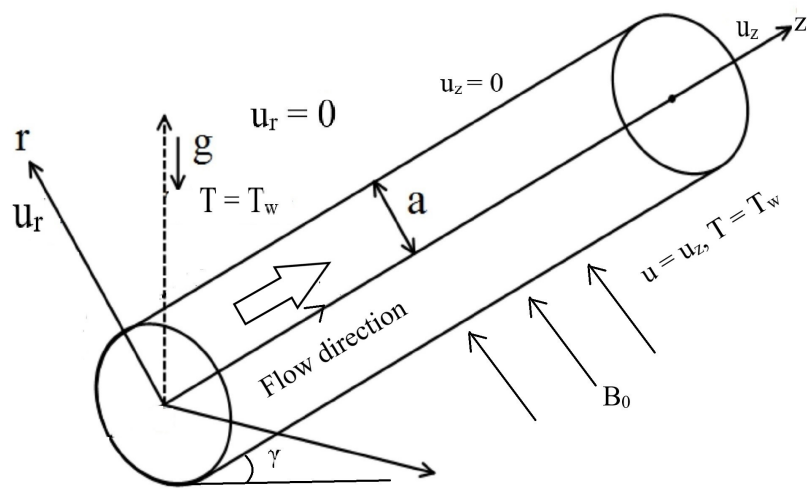

Fig. 1. Flow geometry and coordinate system

The above assumptions, together with boundary layer approximations, the model equations to the problem under study are given as: 


$$
\begin{gathered}
\frac{\partial\left(r u_{r}\right)}{\partial r}+\frac{\partial\left(r u_{z}\right)}{\partial z}=0 \\
u_{r} \frac{\partial u_{z}}{\partial r}+u_{z} \frac{\partial u_{z}}{\partial z}=\nu\left[\frac{1}{r} \frac{\partial}{\partial r}\left(r \frac{\partial u_{z}}{\partial r}\right)+\frac{\partial^{2} u_{z}}{\partial z^{2}}\right]+ \\
{\left[g \beta_{t}\left(T-T_{\infty}\right)\right] \cos \gamma-\sigma \frac{B_{0}^{2} u_{z}}{\rho}} \\
u_{r} \frac{\partial T}{\partial r}+u_{z} \frac{\partial T}{\partial z}=\frac{k}{\rho C_{p}}\left[\frac{1}{r} \frac{\partial}{\partial r}\left(r \frac{\partial T}{\partial r}\right)+\frac{\partial^{2} T}{\partial z^{2}}\right]+\frac{\mu}{\rho C_{p}}\left(T_{w}-T\right)
\end{gathered}
$$

where $u_{r}$ and $u_{z}$ are the velocity components in the $\mathrm{r}$ and $\mathrm{z}$ directions, $\rho$ is the density of the fluid, $\mu$ is the dynamic viscosity of the fluid, $\nu$ is the kinematic viscosity and is given by $\nu=\frac{\mu}{\rho}, k$ is the thermal conductivity of the fluid, $C_{p}$ is the specific heat of the fluid, $T_{w}$ is the cylinder wall temperature, $a$ is the radius of the cylinder, $\sigma$ is the electrical conductivity, $\mathrm{g}$ is the acceleration due to gravity, $\beta_{t}$ is the thermal expansion coefficient, $\mathrm{T}$ is the temperature of the fluid, $T_{\infty}$ is the temperature outside the cylinder and $\gamma$ is the angle of inclination of the cylinder measured from the vertical axis The associated boundary conditions are given by:

$$
u_{r}=0, u_{z}=0, T=T_{w} \text { on } r=a
$$

and,

$$
u_{z} \rightarrow 0, \quad T \rightarrow T_{\infty}, \quad r \rightarrow \infty
$$

1) Non-dimensionalisation of the model equations: As the fluid moves along the z-axis, the velocity tends to be zero, hence the terms $\frac{\partial^{2} u_{r}}{\partial z^{2}}, \frac{\partial^{2} u_{z}}{\partial z^{2}}$ and $\frac{\partial^{2} T}{\partial z^{2}}$ can be ignored. We then non-dimensionalise the remaining terms in the equations 2 and 3 using the following non-dimensional parameters:

$$
\begin{aligned}
& u_{r}=u_{r}^{*} U_{0}, \quad u_{z}=u_{z}^{*} U_{0}, \quad R e=\frac{a U_{0}}{\nu}, r=r^{*} a \\
& \nu=\frac{\mu}{\rho}, \quad N u=\frac{a^{2}}{C p}, \quad G r=\frac{g \beta_{t}\left(T_{w}-T_{\infty}\right)}{U_{0}^{2}} \\
& H a^{2}=\frac{B o^{2} a^{2} \sigma}{\mu}, \text { and } \\
& \theta(\eta)=\frac{T-T_{\infty}}{T_{w}-T_{\infty}}
\end{aligned}
$$

where:

$U_{0}$ is the reference velocity, $a$ is the characteristic length from centre of cylinder, $R e$ is the Reynolds number, $N u$ is the Nusselt number, $\mathrm{Gr}$ is the Grashof number, $\mathrm{Ha}$ is the Hartmann number, $\theta$ is the dimensionless temperature.

Note that quantities with superscript stars are dimensionless quantities.

From equation 2:
Similarly, expressions for the remaining terms in equations 2 , and 3 are worked out then multiply the momentum and energy equations by $\frac{a}{U_{0}^{2}}$ and $\frac{a}{U_{0} T_{w}-T_{\infty}}$ respectively.

Neglecting the *'s and with appropriate substitutions of Re, $\mathrm{Ha}, \mathrm{Nu}, \mathrm{Gr}$, the equations 2 and 3 metamorphoses to;

$$
\begin{gathered}
u_{r} \frac{\partial u_{z}}{\partial r}+u_{z} \frac{\partial u_{z}}{\partial z}=\frac{H a^{2}}{R e} u_{z}+\frac{1}{R e}\left[\frac{1}{r} \frac{\partial u_{z}}{\partial r}+\frac{\partial^{2} u_{z}}{\partial r^{2}}\right]+ \\
\operatorname{Gr} \theta \sin \gamma \\
u_{r} \frac{\partial \theta}{\partial r}+u_{z} \frac{\partial \theta}{\partial z}=\frac{1}{\operatorname{RePr}}\left[\frac{1}{r} \frac{\partial \theta}{\partial r}+\frac{\partial^{2} \theta}{\partial r^{2}}\right]+\frac{N u}{R e} \theta
\end{gathered}
$$

2) Similarity transformation: The similarity transformation converts the non-linear partial differential equations into the set of nonlinear ordinary differential equations. Define a 2-D stream function, $\psi(r, z)$ by;

$u_{r}=-\frac{1}{r} \frac{\partial \psi}{\partial z}=\frac{a}{2 r} \sqrt{\frac{U_{0} \nu}{z}}\left(\eta f^{\prime}-f\right)$ and $u_{z}=\frac{1}{r} \frac{\partial \psi}{\partial r}=U_{0} f^{\prime}$

in which the equation continuity is satisfied identically. We now introduce the following non- dimensional similarity variables to obtain a similarity solution:

$$
\begin{aligned}
& \eta(r, z)=\frac{r^{2}-a^{2}}{2 a} \sqrt{\frac{U_{0}}{\nu z}}, \quad \psi(r, z)=\sqrt{\nu U_{0} z}(a f(\eta)) \quad \text { and } \\
& \theta(\eta)=\frac{T-T_{\infty}}{T_{w}-T_{\infty}}
\end{aligned}
$$

Hence $\eta$ is a similarity variable, $U_{0}$ is the reference velocity, $\theta(\eta)$ is the non-dimensional temperature, and $f^{\prime}$ is the velocity of the fluid.

The above variables are used to convert the partial differential equations, equations 4 and 5 into a system of ordinary differential equations of order 3 and 2 respectively. We get the nonlinear system of ordinary differential equations as shown below:

$$
\begin{array}{r}
\frac{1}{\nu R e}(4 M \eta+2) f^{\prime \prime \prime}-\frac{1}{R e}(4 K M) f^{\prime \prime}+f f^{\prime \prime} \\
+\frac{2 K H a^{2}}{R e} f^{\prime} \\
+2 G r \theta \sin \gamma=0 \\
\frac{4 M \eta+2}{\nu \operatorname{RePr}} \theta^{\prime \prime}+\frac{4 K M}{\operatorname{RePr}} \theta^{\prime}+f \theta^{\prime}+\frac{2 K N u}{R e} \theta=0
\end{array}
$$

With the following boundary conditions:

$$
f(0)=0, \quad f^{\prime}(0)=0, \quad f(\eta) \rightarrow 0, \quad \text { as } \quad \eta \rightarrow \infty, \quad \theta(0)=1,
$$

$$
f^{\prime} \rightarrow 0, \quad \theta \rightarrow 0, \quad \text { as } \quad \eta \rightarrow \infty
$$

where prime indicates the differentiation with respect to $\eta$.

$$
u_{r} \frac{\partial u_{r}}{\partial r}=\frac{U_{0}^{2}}{a} u_{r}^{*} \frac{\partial u_{r}^{*}}{\partial r^{*}}
$$




\section{B. Method of Solution- Shooting method}

The shooting technique is very key when solving the boundary problems. This is a method that converts a boundary value ordinary differential equation into a system of ordinary differential equations of order one which are then solved as Initial value problem (IVP). We evaluate the solution of the IVP obtained at the second boundary point and then compare its value with the actual boundary value provided. An attempt is made to vary the assumed initial condition iteratively until the required boundary conditions of the solution are Satisfied. The main task is to solve the equations as IVPs.

We begin by letting

$$
\begin{array}{cr}
f^{\prime}=w=g_{1}(\eta, f, w, z) & f(0)=0 \\
w^{\prime}=z=g_{2}(\eta, f, w, z) & w(0)=0 \\
z^{\prime}= & \frac{4 \nu K M}{4 M \eta+2} z-\frac{\nu R e}{4 M \eta+2} f z-\frac{2 \nu K H a^{2}}{4 M \eta+2} w- \\
& \frac{2 \nu \operatorname{Re} G r \operatorname{Sin} \gamma}{4 M \eta+2}=g_{3}(\eta, f, w, z) \\
z(0)=?
\end{array}
$$

Similarly, Equation 7 is converted into initial value problem as shown below:

$$
\begin{array}{r}
t^{\prime}=p=g_{4}(\eta, t, p) \\
p^{\prime}=-\frac{4 \nu K M}{4 M \eta+2} t-\frac{\nu \operatorname{RePr} f}{4 M \eta+2} t-\frac{2 \nu K N u \operatorname{Pr}}{4 M \eta+2} \theta=g_{5}(\eta, t, p) \\
p(0)=?
\end{array}
$$

TABLE I

EFFECTS OF $\gamma$, HARTMANN AND GRASHOFF VALUES ON VELOCITY

PROFILE
The resulting initial value problems in each case are further solved by applying the shooting technique [11], which incorporates the Runge-Kutta fourth order method.

\section{RESUlts AND Discussion}

The influence of the key physical parameters under investigation on the velocity fields are shown in Figures 2 to 4 .

Figure 2 shows how $\gamma$ affects the horizontal velocity field. This parameter has significant influence within the dynamic area around the surface. Away from this dynamic area, we note that the velocity field rises as $\gamma$ decreases. The effect of free convection parameter on the horizontal velocity is also displayed in Figure 3. It can be observed that an increase in the Grashoff number decreases the velocity boundary layer. Hence, this causes the subsequent decrease of the fluid velocity along horizontal flow. The transverse velocity field is also affected by the Magnetic parameter as shown in Figure 4. This velocity field is significantly influenced by varying the Hartmann number within the dynamic area. However, away from this dynamic area, the fluid tends to the tangential position because as Hartmann number increases, the velocity decreases.

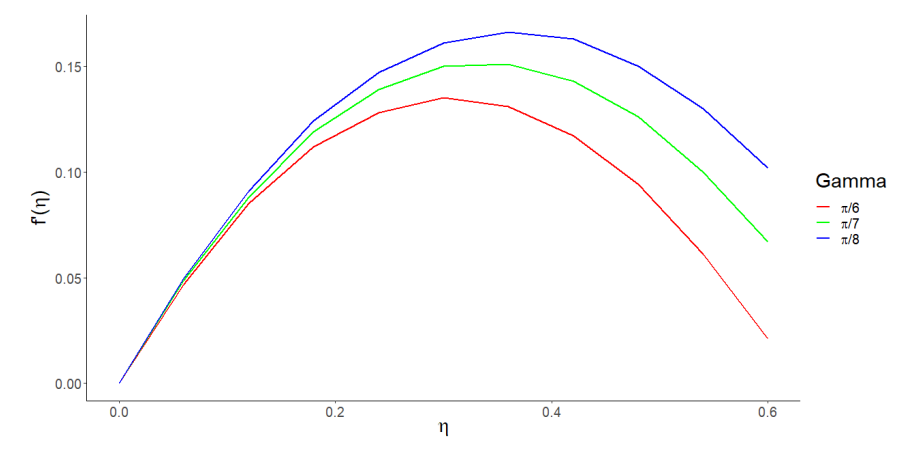

Fig. 2. Effect of $\gamma$ on Velocity Profile

\begin{tabular}{|r|rrr|rrr|rrr|}
\hline & \multicolumn{3}{|c|}{$\gamma$ values } & \multicolumn{3}{|c|}{ Hartmann values } & \multicolumn{3}{c|}{ Grashoff values } \\
Zero & $\pi / 6$ & $\pi / 7$ & $\pi / 8$ & 10 & 11 & 12 & 0.5 & 0.8 & 1 \\
\hline $\mathbf{0 . 0 0}$ & 0.000 & 0.000 & 0.000 & 0.000 & 0.000 & 0.000 & 0.000 & 0.000 & 0.000 \\
$\mathbf{0 . 0 6}$ & 0.047 & 0.049 & 0.050 & 0.048 & 0.050 & 0.049 & 0.056 & 0.052 & 0.049 \\
$\mathbf{0 . 1 2}$ & 0.085 & 0.088 & 0.091 & 0.086 & 0.089 & 0.088 & 0.104 & 0.094 & 0.088 \\
$\mathbf{0 . 1 8}$ & 0.112 & 0.119 & 0.124 & 0.116 & 0.117 & 0.115 & 0.143 & 0.126 & 0.115 \\
$\mathbf{0 . 2 4}$ & 0.128 & 0.139 & 0.147 & 0.136 & 0.135 & 0.131 & 0.174 & 0.148 & 0.131 \\
$\mathbf{0 . 3 0}$ & 0.135 & 0.150 & 0.161 & 0.146 & 0.141 & 0.135 & 0.197 & 0.160 & 0.135 \\
$\mathbf{0 . 3 6}$ & 0.131 & 0.151 & 0.166 & 0.146 & 0.137 & 0.128 & 0.210 & 0.161 & 0.128 \\
$\mathbf{0 . 4 2}$ & 0.117 & 0.143 & 0.163 & 0.137 & 0.122 & 0.111 & 0.215 & 0.153 & 0.111 \\
$\mathbf{0 . 4 8}$ & 0.094 & 0.126 & 0.150 & 0.119 & 0.096 & 0.083 & 0.213 & 0.135 & 0.083 \\
$\mathbf{0 . 5 4}$ & 0.061 & 0.100 & 0.130 & 0.091 & 0.060 & 0.045 & 0.202 & 0.108 & 0.045 \\
$\mathbf{0 . 6 0}$ & 0.021 & 0.067 & 0.102 & 0.054 & 0.015 & 0.002 & 0.184 & 0.073 & 0.002 \\
\hline
\end{tabular}




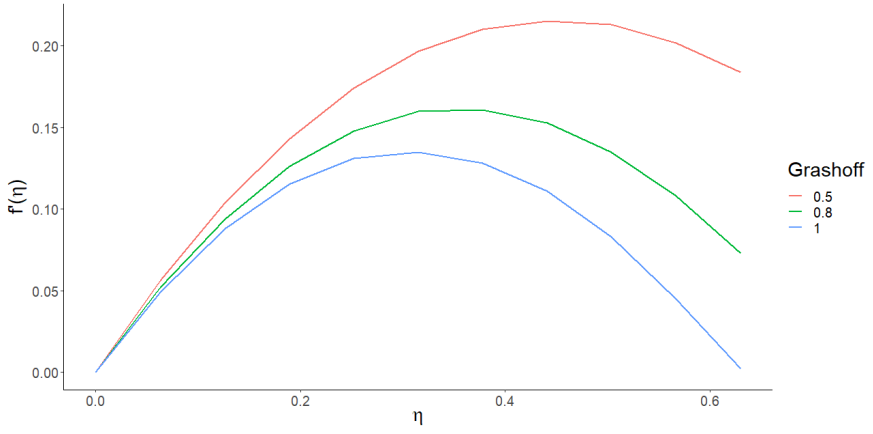

Fig. 3. Effect of Gr on Velocity Profile

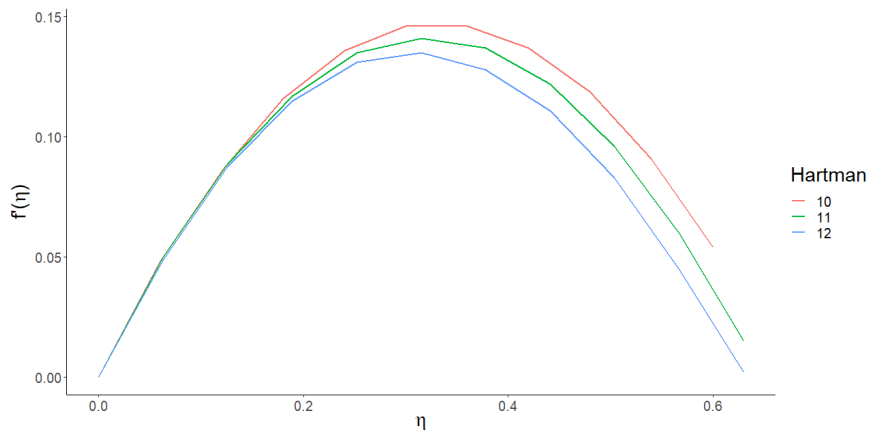

Fig. 4. Effect of Ha on Velocity Profile

\section{CONCLUSION}

The study focused on the numerical solution for steady Magnetohydrodynamic flow along an inclined cylinder under the influence of varied magnetic field. The study found out that optimal inclination plays a key role since it affects flow velocity. The rate of transfer also reduces considerably by increasing the Grasshoff number. Further, an increase in magnetic parameter decreases the velocity. The physics of flow through inclined cylinders can be applicable in a number of engineering and scientific situations with the help of the present model. The results of the study may be useful for the different model investigations. The results of the current investigation is of great importance in various areas of science and technology in which optimal inclination and free convection are utilized.

\section{REFERENCES}

[1] Abdelmeguid A.M and Spalding D.B Turbulent flows and heat transfer in pipes with buoyancy effects, A Journal of fluid mechanics, 1979;94(2):383-400.

[2] Alam M.S et al. MHD free convective heat and mass transfer flow past an inclined surface with heat generation, Thammasat international journal of science and technology , 2006;11 (1): 1-9.

[3] Anjali S.P. et al.Effect of magnetic field on Blasius and Sakiadis flow of nano fluids passed an inclined plate, Journal of Taibah university for science, 2017;5(4):1-27.

[4] Babatunde A. and Peter B. Magnetohydrodynamic convection fluid and heat transfer in an inclined micro porous channel, Non linear Engineering,2019;8(1):755-763

[5] Chen C-H et al. Heat and mass transfer in MHD flow by natural convection from a permeable inclined surface with variable wall temperature and concentration, Acta mechanical journal ,2004;71(172):219-235.

[6] Gasljevic K. et al. Buoyancy effects on heat transfer and temperature profile in horizontal pipe flow of drag reducing fluids, International Journal of heat and mass transfer, 2000;43(23):4267-4274.

[7] Hasnain et al.Buoyant displacement flow of immiscible fluid in inclined pipe, Journal of fluid mechanics, 2017;29(5):661-687.

[8] Hartmann J.and Lazarus F.Experimental investigation on the flow of mercury in a homogenous magnetic field, Mercury dynamics, 1937;15(7):3367-3380.

[9] Majid et al. Effects of buoyancy on heat transfer in supercritical water flow in a horizontal round tube, Journal of heat transfer, 2005;127(8):897902.

[10] Maruf Hasan et al. MHD free convection flow passed an inclined stretching sheet with considering viscous dissipation and radiation, Open journal of fluid dynamics , 2017;7(2):152-168.

[11] Manyonge A.W et al.Numerical solution of nonlinear boundary value problems of ordinary differential equations using shooting technique, Journal of innovative Technology and education, 2017;4(1):29-36.

[12] Opiyo R. et al. Numerical computation of steady buoyancy driven MHD heat and mass transfer past an inclined infinite plate with sinusoidal surface boundary conditions, Applied mathematical sciences, 2017;11(15):711-721.

[13] S.Shateyi and G.T.Marewo. A numerical approach for the laminar boundary layer flow and heat transfer along a stretching cylinder embedded in a porous medium with variable thermal conductivity, A Journal of Applied mathematics,2013; ID576453.

[14] Shikha K. et al. Numerical investigation of nanofluid heat transfer in an inclined stretching cylinder under the influence of suction/injection and viscous dissipation, International journal of nano mechanics science and technology, 2019;10(1):29-49.

[15] Swati M. MHD boundary layer slip flow along a stretching cylinder,Ains Shams Engineering Journal, 2013;4,317-324.

[16] Varma S. et al. Diffusion-thermo and aligned magnetic field effects on free convection on flow passed inclined porous plate with first order chemical reaction, Journal of electrical and electronic engineering, 2017;12(3):25-33.

[17] Yeng-Yung T.and Sheu-Jang S. Effects of buoyancy and orientation on the flow in a duct preceded with a double step expansion,International journal of heat and mass transfer, 1998;41(17):2687-2695. 